\title{
Repurposing staples for viruses: applying peptide design to RSV prophylaxis
}

\author{
Sarah P. Katen ${ }^{1,2}$ and Terence S. Dermody $y^{1,2,3}$
}

\author{
1Department of Pediatrics, '2Department of Pathology, Microbiology, and Immunology, and \\ ${ }^{3}$ Elizabeth B. Lamb Center for Pediatric Research, Vanderbilt University School of Medicine, Nashville, Tennessee, USA.
}

\begin{abstract}
Respiratory syncytial virus (RSV) is responsible for lower respiratory tract infections and annually results in 200,000 deaths worldwide. Despite the burden of RSV-associated disease, treatments and preventative measures are limited. In this issue of JCI, Bird and colleagues describe their work using a peptide stapling technique that allowed synthesis of a stable peptide mimic of a portion of the RSV fusion protein. Pretreatment of cells with the stable peptide effectively blocked virus entry. When introduced into mice prior to RSV exposure, the peptide produced a substantial prophylactic effect. This work provides a new way forward in RSV prevention.
\end{abstract}

\section{Respiratory syncytial virus treatment: what's left that we haven't tried?}

Respiratory syncytial virus (RSV) causes nearly 64 million lower respiratory tract infections each year, most commonly striking the very young and very old and resulting in significant mortality in these age groups (1). The status of RSV as a major public health threat has been a primary motivator in the development of antiviral treatment and prophylactic strategies; however, progress on this front has been modest. The current gold standard for at-risk infants is prophylactic administration of the monoclonal antibody palivizumab, but this approach yields only modest efficacy (2). Treatment with the nucleoside analog ribavirin may have utility in certain situations, but its use is limited by toxicity and cost (3). Thus far, there are no FDA-approved vaccines or effective RSV-specific treatments (4).

Recent efforts toward prevention and treatment of RSV disease have focused on the RSV trimeric fusion protein (RSV-F). Structural studies show that RSV-F has the classic fusogenic mechanism of two 3-helix bundles (3-HBs), which undergo structural rearrangement and association around a hairpin turn to bring the viral and cellular membranes in close apposition to allow membrane fusion (Figure 1A and refs. 5, 6). Numerous small-molecule

Conflict of interest: The authors have declared that no conflict of interest exists.

Citation for this article: J Clin Invest. 2014; 124(5):1889-1891. doi:10.1172/JCI75797. and peptide inhibitors have been used to impede RSV fusion in cells and thus block viral entry $(7,8)$; however, none of the current small-molecule antiviral compounds designed to inhibit RSV infection have progressed to clinical testing. Perhaps more encouragingly, a truncated form of RSV-F trapped in the prefusion state and conjugated to immunostimulatory peptides or particles elicits potent neutralizing antibody responses $(9,10)$. Despite these developments, further avenues for RSV prophylaxis and therapy continue to be explored. In this issue of JCI, Bird and colleagues describe a new take on peptidebased prophylaxis and inhibition of RSV infection (11) that has the potential to serve as an effective prophylactic strategy for at-risk populations and may be applicable for other viral pathogens.

\section{Stapled peptides: a chemist's answer to a virologist's question}

Small molecules have been useful as antiviral agents due to their stability, capacity to be synthesized in large amounts, and ease of delivery to patients. More recently, monoclonal antibodies have been used as antivirals and exhibit strong and specific antiviral activity with few adverse effects and the added benefit of serving as prophylactic agents for at-risk populations. Despite their therapeutic potential, the identification and preparation of neutralizing antibodies for human use is time consuming, and production of antibodies in sufficient quantities for treatment is expensive. One therapeutic option that has lagged behind more traditional approaches is the development of antiviral peptides. Long the domain of chemists and structural biologists, peptides have been underrepresented as anti-infective therapeutics due to their instability, susceptibility to proteases, and challenges regarding delivery to the infected site. However, a new technique for engineering peptide stability may hold the solution to the pharmacologic problems that have sidelined peptide antivirals.

Bird et al. adapted the technique of hydrocarbon "stapling" to their choice of antiviral peptide to generate stabilized $\alpha$-helices of RSV-F (termed SAH-RSVF; ref. 11). The peptide in question is a short $\alpha$-helical mimic of one of the helices involved in formation of the RSV-F fusogenic 6-HB. By binding to the prefusogenic 3-HB form of RSV-F, the peptide blocks formation of the fusogenic state and thus inhibits fusion and viral entry (Figure 1B). The peptide-based, HIV-1-specific antiviral enfuvirtide potently blocks viral entry via the same mechanism (12). There have been attempts to recapitulate this success with RSV, but the RSV-specific peptides tend to form random coils in solution, which decreases peptide stability and activity (13). Bird and colleagues adapted a biochemical technique to avoid these pitfalls and introduced atypical amino acids with olefin tethers at positions in the engineered peptides exposed to the outer face of the peptide helix (11). These hydrocarbon tethers were chemically cross-linked, which effectively staples the turns of the helix together and clamps them into an $\alpha$-helical structure (Figure 1B, inset, and ref. 14). This modification increases the stability of SAH-RSVF by locking it into a constrained $\alpha$-helix, mimicking the bioactive conformation and reducing susceptibility of the peptide to proteolytic attack. Thus, the use of a chemical trick to induce and stabilize the $\alpha$-helical structure of a short peptide has overcome a longstanding obstacle in the development of peptides for biological applications. 
A
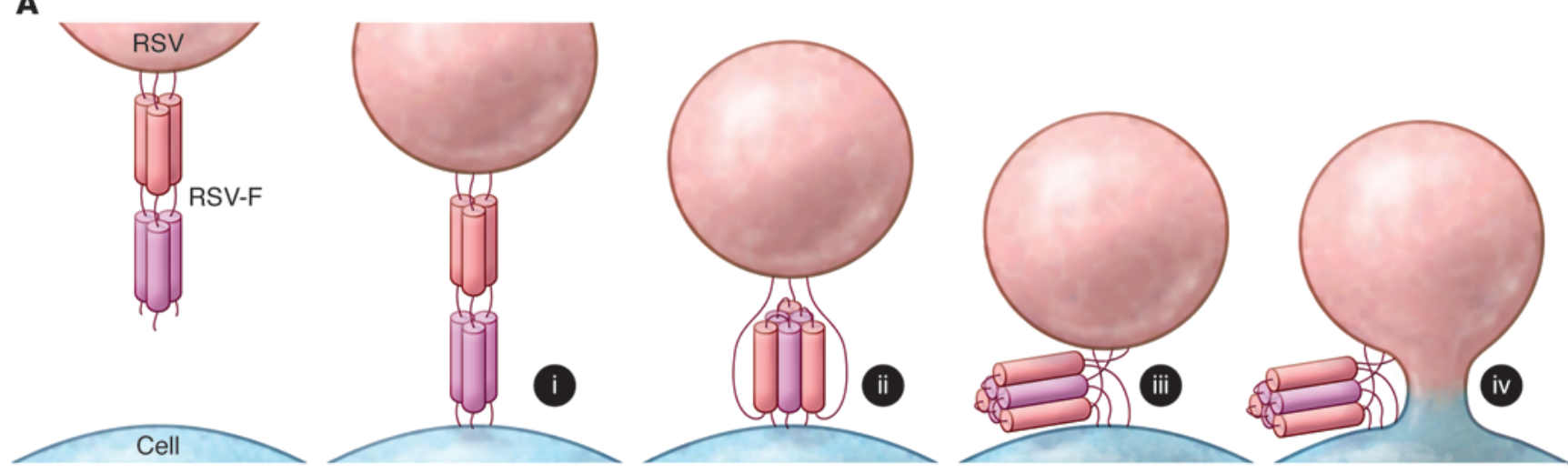

B
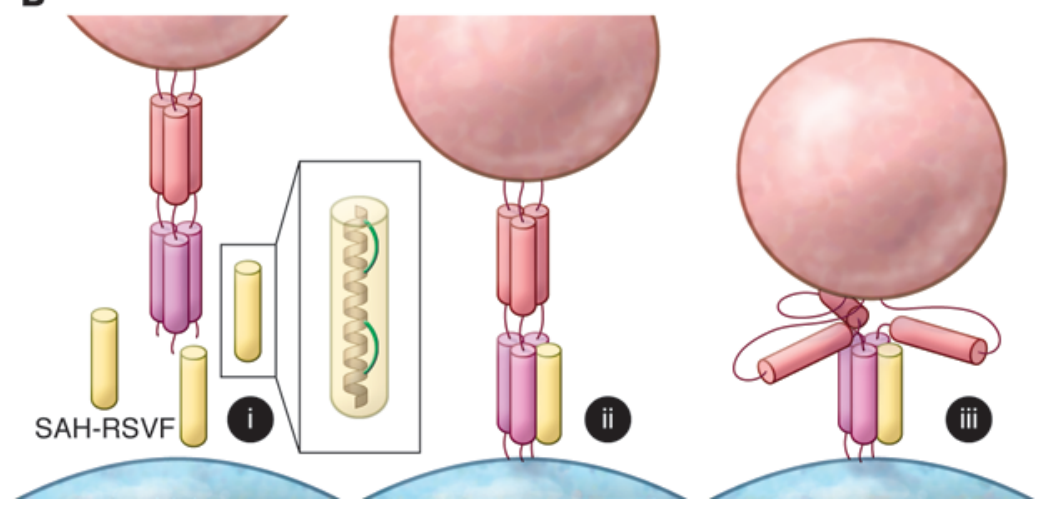

Figure 1

Inhibition of RSV fusion with stapled peptides. (A) RSV fusion. Prefusion RSV-F, consisting of two 3-HBs (red and pink cylinders), inserts into the cell membrane (i). Structural rearrangement of RSV-F (ii) results in formation of a 6-HB that brings the two membranes together (iii) for fusion via a hemifusion intermediate (iv). (B) Inhibition by stapled peptides. The antiviral peptide SAH-RSVF (yellow cylinder) is an $\alpha$-helical peptide (gray, inset) stapled with cross-links that span two helical turns (green, inset). The peptide mimics one of the fusion helices (i) and binds to RSV-F in the prefusion state (ii), blocking formation of the fusogenic $6-\mathrm{HB}$ and preventing membrane fusion (iii).

\section{Viral infection prophylaxis: are peptides back on the table?}

The question of efficacy for antiviral peptides often comes down to the issues of stability and delivery. Will the peptide assume the required conformation and resist degradation once introduced into an infected host, and will it reach the site of infection? In answer to these pharmacological questions, Bird et al. showed that their stapled peptides display a marked antiviral effect when administered to mice prior to RSV infection (11). By instilling SAH-RSVF intranasally, a peptide barrier is effectively created at the RSV entry point. Infection in the nares was markedly reduced by pretreatment with SAH-RSVF, which indicates that the peptides are stable and functionally active. Perhaps more impressive was the observation that peptide activity was not limited to the site of administration. RSV spread to the lower respiratory tract and caused substantial lower respiratory dis- ease, but during the course of their experiments, the authors found that the intranasally administered peptide also markedly decreased pulmonary RSV infection (11). Incorporation of the hydrocarbon staples appears to have stabilized SAH-RSVF to the point that it can travel to a distant site of infection without compromising its biological potency.

The initial route of administration, however, sidesteps the question of how to effectively deliver a peptide to a systemic site of infection. RSV has the "advantage" of being a respiratory tract virus for which the route of entry is a convenient site for peptide administration. For viruses with alternative routes of entry or for persons already infected, administration at the entry site may not be effective. To circumvent this problem, Bird et al. turned to nanotechnology to further augment their antiviral treatment, generating a chitosan-based nanoparticle to encapsu- late SAH-RSVF with the goal of improving efficacy and delivery (11). This technique has been shown to be effective with both small-molecule and nucleic acid-based treatments $(15,16)$. The nanoparticleencased peptides were administered intratracheally, delivering the SAH-RSVF peptides directly to the site of pulmonary RSV infection, rather than just at the point of entry. Peptide incorporation into the nanoparticle increased the extent and uniformity of delivery, which led to a striking improvement in viral prophylaxis over peptide alone (11).

\section{Conclusions and future directions}

In today's multidisciplinary research environment, the traditional divisions among the basic sciences have become blurred, providing exciting opportunities to combine different approaches, improving old techniques for disease therapeutics and developing new ones. The study by Bird 
et al. (11) is an excellent example of scientists pushing past the traditional limits of their discipline to take advantage of biology, chemistry, and nanotechnology and overcome the shortcomings of clinical applications of antiviral peptides. While the authors focused on the use of stabilized peptides and nanoparticle delivery as prophylactic measures, their cell-based work demonstrating robust inhibition of RSV fusion and entry (11) suggests that the peptides could be adapted as antiviral therapeutics for persons with RSV infection. Moreover, the formation of hairpin-linked 6 -HBs is a classic hallmark of many fusogenic enveloped viruses, including Ebola virus, HIV, and SARS coronavirus, which all bring viral and cellular membranes together via formation of a $6-\mathrm{HB}$ in the same fashion as RSV (17). It is possible that the present findings made with RSV could be adapted to combat a host of pathogenic human viruses, both as prophylactic agents and as antiviral treatments.

\section{Acknowledgments}

The authors were supported by grants from the NIH (T32 AI095202 to S.P. Katen; R01 AI076983 to T.S. Dermody).
Address correspondence to: Terence S. Dermody, Elizabeth B. Lamb Center for Pediatric Research, Vanderbilt University School of Medicine, D7235 MCN, 1161 21st Avenue South, Nashville, Tennessee 37232, USA. Phone: 615.343.9943; Fax: 615.343.7659; E-mail: terry.dermody@vanderbilt.edu.

1. World Health Organization. Initiative for Vaccine Research, Acute respiratory infections. WHO Web site. http://apps.who.int/vaccine_research/diseases/ ari/en/index.html. Updated September 1, 2009. Accessed March 10, 2014.

2. Andabaka T, Nickerson JW, Rojas-Reyes MX, Rueda JD, Bacic Vrca V, Barsic B. Monoclonal antibody for reducing the risk of respiratory syncytial virus infection in children. Cochrane Database Syst Rev. 2013;4:CD006602.

3. Hall CB, et al. Respiratory syncytial viral infection in children with compromised immune function. N Engl J Med. 1986;315(2):77-81.

4. Haynes LM. Progress and challenges in RSV prophylaxis and vaccine development. J Infect Dis. 2013; 208(suppl 3):S177-S183.

5. McLellan JS, Yang Y, Graham BS, Kwong PD. Structure of respiratory syncytial virus fusion glycoprotein in the postfusion conformation reveals preservation of neutralizing epitopes. J Virol. 2011; 85(15):7788-7796.

6. McLellan JS, et al. Structure of RSV fusion glycoprotein trimer bound to a prefusion-specific neutralizing antibody. Science. 2013;340(6136):1113-1117.

7. Douglas JL, et al. Inhibition of respiratory syncytial virus fusion by the small molecule VP-14637 via specific interactions with F protein. J Virol. 2003; 77:5054-5064.
8. Razinkov V, Gazumyan A, Nikitenko A, Ellestad G, Krishnamurthy G. RFI-641 inhibits entry of respiratory syncytial virus via interactions with fusion protein. Chem Biol. 2001;8(7):645-659.

9. Garg R, Latimer L, Simko E, Gerdts V, Potter A, van den Hurk S. Induction of mucosal immunity and protection by intranasal immunization with a respiratory syncytial virus subunit vaccine formulation. J Gen Virol. 2014;95(pt 2):301-306.

10. Rigter A, et al. A protective and safe intranasal RSV vaccine based on a recombinant prefusion-like form of the F protein bound to bacterium-like particles. PLoS One. 2013;8(8):e71072.

11. Bird GH, et al. Mucosal delivery of a double-stapled RSV peptide prevents nasopulmonary infection. J Clin Invest. 2014;124(5):2113-2124.

12. Kilby JM, et al. Potent suppression of HIV-1 replication in humans by T-20, a peptide inhibitor of gp41mediated virus entry. Nat Med. 1998;4(11):1302-1307.

13. Lambert DM, et al. Peptides from conserved regions of paramyxovirus fusion $(\mathrm{F})$ proteins are potent inhibitors of viral fusion. Proc Natl Acad Sci US A. 1996;93(5):2186-2191.

14. Schafmeister C, Po J, Verdine GL. An all-hydrocarbon cross-linking system for enhancing the helicity and metabolic stability of peptides. J Am Chem Soc. 2000;122(24):5891-5892.

15. Luo Y, Wang Q. Recent development of chitosanbased polyelectrolyte complexes with natural polysaccharides for drug delivery. Int J Biol Macromol. 2014;64C:353-367.

16. Menon JU, Ravikumar P, Pise A, Gyawali D, Hsia CC, Nguyen KT. Polymeric nanoparticles for pulmonary protein and DNA delivery. Acta Biomaterialia. 2014;pii:S1742-7061(14)00052-X.

17. Melikyan GB. Common principles and intermediates of viral protein-mediated fusion: the HIV-1 paradigm. Retrovirology. 2008;5:111.

\section{A VISTA on PD-1H}

\section{Yang Liu}

Centers for Cancer and Immunology Research, Children's Research Institute, Children's National Medical Center, Washington, DC, USA.

\begin{abstract}
Three years ago, two research groups independently identified a previously undescribed $\mathrm{T}$ cell cosignaling molecule; one referred to it as V-domain Ig suppressor of $T$ cell activation (VISTA), and the other used the term programmed death-1 homolog (PD-1H). Recombinant and ectopically expressed PD-1H functions as a coinhibitory ligand for $\mathrm{T}$ cell responses. However, the function of endogenous PD-1H is not clear. In this issue of the JCI, Flies and colleagues demonstrate that endogenous PD-1H on both $T$ cells and APCs serves as a coinhibitory molecule for $T$ cell activation and provide further support for targeting PD-1H as a therapeutic strategy for transplantation and cancers.
\end{abstract}

Life is never dull, especially if you are interested in $\mathrm{T}$ cell cosignaling molecules. The identification of new members of this family can lead not only to new paradigms in immune recognition, but can also affect the treatment of cancer and autoimmune

Conflict of interest: The author has declared that no conflict of interest exists.

Citation for this article: J Clin Invest. 2014; 124(5):1891-1893. doi:10.1172/JCI75798. diseases (1-4). Among the newest additions to the $\mathrm{T}$ cell cosignaling family is an immunoglobulin superfamily member that bears two different names due to its independent discoveries three years ago: $\mathrm{V}$-domain Ig suppressor of $\mathrm{T}$ cell activation (VISTA) and programmed death-1 homolog (PD-1H).

Wang et al. named this molecule VISTA (5), based on significant sequence homology with $\mathrm{B} 7$ homologue 1 (B7H1) and the ability of recombinant VISTA-Fc protein and ectopic expression of VISTA on APCs or tumor cells to inhibit $\mathrm{T}$ cell proliferation. In an independent study, Flies and colleagues identified the same molecule and named it PD-1H due to greater overall sequence and gene structure similarities with programmed death-1 (PD-1) (6).

\section{Same target, different results}

Remarkably, the antibodies produced against the molecule by Wang and colleagues and Flies et al. had starkly different effects. Whereas Wang et al. found that their anti-VISTA antibody exacerbated EAE, Flies et al. found that their antiPD-1H antibody suppressed graft-versushost diseases (GVDH) to such an extent that fully allogeneic bone marrow chimera mice (BALB/c to lethally irradiated C57BL/6 mice) could be obtained following a single dose of anti-PD-1H antibody 